\title{
Spatial selectivity in visual search
}

\author{
JAMES E. HOFFMAN and BILLIE NELSON \\ University of Delaware, Newark, Delaware 19711
}

\begin{abstract}
To what extent does successful search for a target letter in a visual display depend on the allocation of attention to the target's spatial position? To investigate this question, we required subjects to discriminate the orientation of a briefly flashed U-shaped form while searching for a target letter. Performance operating characteristics (POCs) were derived by varying the relative amounts of attention subjects were to devote to each task. Extensive tradeoffs in performance were observed when the orientation form and target letter occurred in nonadjacent display positions. In contrast, the tradeoff was much more restricted when the two targets occurred in adjacent positions. These results suggest that the interference between simultaneous visual discriminations depends critically on their separation in visual space. Both visual search and form discrimination require a common limited-capacity visual resource.
\end{abstract}

This paper is concerned with the question of whether successful detection of a target letter in a visual array of letters depends on the allocation of attention to the spatial region containing the target. We introduce a method for measuring the location of a subject's visual attention and show that allocation of attention to visual targets is a component of the search process. Correct target detections are associated with allocation of attention to the spatial region containing the target, whereas incorrect target detections are associated with allocation of attention to nontarget areas.

\section{Spatial Selective Attention}

Acquisition of information from text or pictures requires a series of saccadic eye movements in which the fovea is brought to bear on different parts of the input to provide high resolution processing of local details. A similar mechanism appears to operate within a single fixation; observers can use an attentional mechanism to selectively "scan" different regions of the input. For example, if one fixates a point on this page, such as the preceding period, one can selectively "read" different letters in the area surrounding fixation. This is a central attentional process that we will refer to as spatial selective attention.

Although the phenomenology of spatial selectivity is compelling, its role in visual information processing is unclear. Consider the case of visual search for

This research was supported by the Personnel and Training Research Programs, Psychological Sciences Division, Office of Naval Research, under Contract N00014-78-C-0762, Contract Authority Identification NR $150-425$. Portions of these data were presented at the 21st annual meeting of the Psychonomic Society, St. Louis, Missouri, November 1980 . The authors would like to acknowledge the hardware and software support provided by the University of Delaware Plato Project. a target in which an observer views a briefly presented array of visual forms such as alphanumeric characters and must indicate whether or not any one of a set of predefined target characters is present in the array. To what extent must the observer shift his/ her attention to each of the display characters to determine whether or not they are targets, and, if such a shift of attention does occur, how similar is it to the process that one employs in "reading" different letters arranged about a point in fixation? Consider, first, the evidence that indicates that spatial selectivity is a component of the search process.

\section{Spatial Selectivity in Visual Search}

A persistent finding in visual search experiments is the display size effect. In general, as the number of nontarget display characters (distractors) increases, target detection accuracy decreases (Estes \& Taylor, 1966; Hoffman, 1978, 1979; Schneider \& Shiffrin, 1977). In addition, target detection latency increases as a linear function of display size, suggesting that display characters are being examined by a serial process (Schneider \& Shiffrin, 1977; Sternberg, Note 1). This serial scanning of display characters is presumably accomplished by the spatial selectivity mechanism.

There is, however, a compelling explanation of the display size effect that does not depend on serial scanning or, indeed, a capacity limitation of any kind. Eriksen and Spencer (1969) and Kinchla (1974) pointed out that a display size effect is predicted even by a model that assumes that classification of display elements is conducted by a parallel, independentchannel, unlimited-capacity process. As the number of display elements increases, so too does the probability that at least one distractor will be mistaken as a target. This increase in "noise" in the decision 
process leads to decreases in detection accuracy. Variants of the "perceptual confusions" model provide a remarkably good quantitative description of a wide variety of search experiments (Eriksen \& Spencer, 1969; Kinchla, 1974; Lappin \& Uttal, 1976).

Although the independent-channels model offers a precise, quantitative description of detection performance in many experiments, there are situations in which its predictions are disconfirmed. Specifically, this model predicts that presentation of the display elements sequentially in time should not improve detection accuracy relative to simultaneous presentation. As long as the total number of potential confusions remains constant, the spatiotemporal aspects of presentation should be unimportant (assuming that peripheral factors such as masking, retinal location, etc., are controlled). Eriksen and Spencer (1969) and Shiffrin and Gardner (1972) confirmed this prediction. Hoffman (1978, 1979), however, found that sequential presentation could produce large increases in detection accuracy relative to simultaneous presentation. The crucial difference between experiments that do and do not find effects of sequential presentation appears to be the kind of training the subjects receive with the memory set. Eriksen and Spencer (1969) and Shiffrin and Gardner (1972) used a training schedule that Schneider and Shiffrin (1977) call consistent mapping (CM), in which target and distractor characters never exchange roles. CM training leads to "automatic detection," and therefore it may not be surprising that sequential presentation does not improve performance. In contrast, varied mapping (VM) training leads to controlled processing, which is characterized as a serial search of the display. A serial search of the display should benefit from sequential presentation.

In summary, the visual search literature suggests that a spatial selection mechanism is a component of controlled processing, while automatic processing does not require spatial selection. It would be desirable to have an independent measure of the location of a subject's attention in visual space. Such a measure would provide a means of verifying the presumably different roles played by spatial selection in controlled and automatic detection.

\section{Measuring the Location of Visual Attention}

Figure 1 shows the proposed method for measuring the location of visual attention during visual search. The observer is required to perform two different tasks. One task is letter search, in which the subject is to determine which of two target letters is present in the display. The second task is to determine the orientation of a briefly flashed U-shaped figure. If correct search trials are the result of the subject's "scanning" the target position, then we would expect that presenting the orientation target in a position adjacent to the target letter would pro-
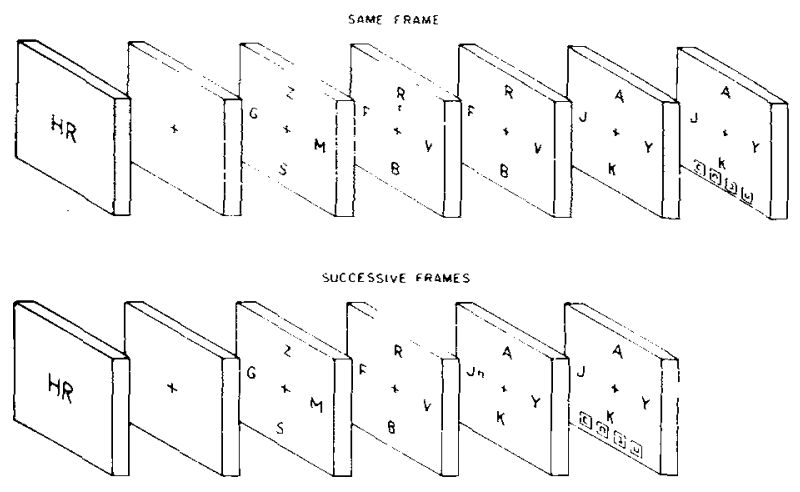

Figure 1. A schematic representation of the sequence of events occurring on each trial. In the same frame condition, the subject was presented with two memory set letters followed by a fixation cross. At trial initiation, a set of premasks was presented for 500 msec followed by a simultaneous onset of the target array and orientation symbol. The orientation symbol terminated prior to offset of the target array. Target array offset was followed by presentation of the postmasks. Following the subject's search task response, a set of response boxes prompted the orientation task response. The successive frame condition was identical except that onset of the orlentation symbol coincided with onset of the postmasks.

duce better orientation discrimination than presenting it in nonadjacent positions. Conversely, if incorrect search trials are the result of the subject's failing to attend to the target letter, orientation accuracy should be superior when the $U$ occurs in positions nonadjacent to the target letter.

These spatial proximity effects are to be expected only if the spatial selectivity occurring during visual search shares important characteristics with the selectivity revealed by experiments that explicitly direct a subject's attention to a location in space. In particular, we are assuming that attention to a display letter will affect processing of adjacent forms. This assumption is clearly supported when attention is directed to a letter by a visual cue. It appears that there is a region approximately $1 \mathrm{deg}$ of visual angle in extent centered on the attended position. Forms falling within this "attentional field" are processed to a higher level than are forms falling in other areas (Eriksen \& Eriksen, 1974; Eriksen \& Hoffman, 1972; Hoffman, 1975).

Attention appears to have a temporal as well as a spatial extent. Selection time appears to be a random variable with a minimum time of $50 \mathrm{msec}$ (Hoffman, 1975 ) and ranging up to some $300 \mathrm{smec}$ (Colegate, Hoffman, \& Eriksen, 1973). In order to encompass this range of times, the orientation symbol occurred in either the same frame as the target letter or the succeeding one.

\section{Performance Operating Characteristics}

The proposed method seeks to evaluate the role of spatial selective attention in visual search by observing how accuracy on an additional task (orienta- 
tion discrimination) is influenced by the distance between the letter target and orientation form. In these circumstances, there is a good chance that our measurement procedure (orientation discrimination) may disturb the task in which we are really interested (visual search). For example, if subjects chose to "concentrate" on the orientation task, we might find that the adjacency of the orientation symbol to the target letter improved search performance, while orientation accuracy was unaffected. In order to assess the interaction of the two tasks across a wide range of strategies, we employed the method of "performance operating characteristics" (POCs) (Kinchla, 1980; Navon \& Gopher, 1979; Sperling \& Melchner, 1978). Subjects were instructed to vary the relative amounts of "attention" to be devoted to the two tasks. For example, they were instructed to "devote $80 \%$ of your attention to the search task and $20 \%$ to the orientation task." The resulting tradeoff in performance between the two tasks across different attention instructions defines a POC.

In spatial attention is a mechanism that both tasks require, and if it is a shareable resource (Navon \& Gopher, 1979) only when forms fall within a single "attentional field," then we should observe quite different POCs for adjacent and nonadjacent targets. Specifically, nonadjacent targets should produce greater tradeoffs in performance than should adjacent targets because nonadjacent targets cannot efficiently share attention.

\section{METHOD}

\section{Subjects}

Subjects were three males and one female, with normal or corrected to normal vision, who were paid for their participation.

\section{Apparatus and Stimuli}

Presentation of visual displays and timing were provided by a PLATO V terminal, which has a plasma panel screen. Timing was provided by the terminal's microprocessor and had a period of approximately $7+1 \mathrm{msec}$. Letters and masks were $.35 \times .27 \mathrm{deg}$ of visual angle in height and width, respectively, and were defined on a $9 \times 7$ dot matrix. Four letters appeared in a circular display with a diameter of $4.27 \mathrm{deg}$ of visual angle. The symbol used for the orientation task was defined on a $5 \times 5$ dot matrix, subtending a visual angle of $.2 \times .2 \mathrm{deg}$, and was always plotted $.17 \mathrm{deg}$ toward the center of the circle from the letter display. Subjects responded by pressing keys on a typewriter-style keyboard.

The luminance of a blank screen was $.2 \mathrm{fL}$, while a fully illuminated screen produced a luminance of $6.5 \mathrm{fL}$.

\section{Procedure}

Each subject served in eight sessions. Each session consisted of five blocks of 64 trials. The display sequence was similar in each block, and the blocks differed only in instructional condition. Before each trial, subjects were shown two letters to associate with two key responses. Each trial display of four letters then contained one of these target letters as well as the orientation form. The orientation form appeared with either the onset of the letter search display (same frame) or onset of the postmasks (successive frames). Subjects were required to press the appropriate key in response to the letter search and then to indicate the symbol's orientation, also by means of the keyboard.
}

In one of the blocks, subjects performed only the letter search task; in another block, only the orientation discrimination task was required. In the remaining three blocks, subjects were asked to divide their attention between the two tasks in one of three ways: $80 \%$ search $20 \%$ orientation, $50 \%$ search $/ 50 \%$ orientation, or $20 \%$ search $/ 80 \%$ orientation. Subjects were told to perform the search task as quickly and accurately as possible, with accuracy stressed over speed. No significant variation in RTs was observed, and they will not be discussed in this report. The order of blocks within a session was random, with the constraint that, across sessions, each block be represented as equally as possible in the ordering.

On each trial, the subject was first presented with the memory set, which remained on view until a keypress initiated the following sequence. A fixation cross appeared in the center of the screen for $1 \mathrm{sec}$, followed by a sequence of three arrays. A typical sequence is shown in Figure 1. A set of four premasks appeared for $500 \mathrm{msec}$ and then was replaced by the target array of letters. The duration of the letter array was dependent on each subject's search performance in preliminary tracking trials. The postmask letters then replaced the target array letters and remained in view until a response occurred. The orientation symbol appeared either at the time of trial array onset (same frame) or with postmask onset (successive frames). The symbol remained on for a duration that was dependent on a second set of preliminary tracking trials performed on the orientation task. The tracking manipulated the display or symbol duration so that a subject's performance would approximate $75 \%$ accuracy on each single task. Each subject was required to perform 24 trials of each task to satisfy this preliminary tracking procedure each session. The letter display duration, averaged across subjects and sessions, was $222 \mathrm{msec}$, with a range for individual subjects of 198 to $235 \mathrm{msec}$. The orientation symbol duration was $105 \mathrm{msec}$ with a range of 53 to $130 \mathrm{msec}$ for individual subjects. At the end of each trial, the subject received feedback concerning the accuracy of response on each task. No RT feedback was provided.

The subject initiated each trial with his/her left hand and indicated which letter of the memory set appeared in the display by pressing the appropriate key with the right hand. In blocks devoted only to the orientation discrimination task, the subject was similarly required to execute a motor response with the right hand. In this instance, the right-hand keypress only brought the display of symbol orientations (with key numbers) to the screen so that an appropriate key could be selected.

A varied mapping procedure was used for the search task. The memory set of two letters and the distractor letters were always taken randomly from the set $[B, D, F, H, N, P, R, V]$. Preand postmask letters were selected randomly without replacement from the remaining 18 letters of the alphabet.

Within each orientation form onset condition, assignment of target letter to positions in the display was random but equally balanced across each of the four positions. The orientation symbol was presented randomly and equally next to the four display positions, with the additional constraint that the probe occur equally in all positions relative to the target location. Within each block, then, the spatial positions of target letter and orientation form were independent.

\section{RESULTS}

If spatial selectivity is a resource utilized in visual search and can be efficiently shared only by forms falling within a restricted attentional field, we should find different performance operating characteristics (POCs) for the case in which targets from both tasks are adjacent to each other relative to when they are nonadjacent. Specifically, the POC for adjacent targets should be closer to the "independent point" in 
which dual-task performance on each task is equivalent to corresponding single-task performance.

Figure 2 shows that these expectations were confirmed. The left panel shows POCs for the case in which the target letter and orientation symbol occurred in nonadjacent display positions. Independent performance of the two tasks would produce a POC at the intersection of a horizontal line through single-task performance of the orientation task (indicated by points on the ordinate) and a vertical line through single-task control performance of the search task (indicated by points on the abscissa). It is clear that the empirical POCs are not located at the independence point, even in the case in which the targets occurred in successive frames. These two tasks are evidently almost totally incompatible.

The nonadjacent POCs are approximately linear, in agreement with the POCs obtained by Sperling and Melchner (1978) for the case of two letter search tasks. Notice that if the same-frame POCs are extrapolated to meet the point at which orientation performance is at single-task levels, the search performance $d^{\prime}$ would be close to zero. In other words, were subjects to allocate $100 \%$ of their attention to the orientation task, they would have little knowledge of the search-task target letter. In trying to extrapolate the POC in the other direction, to meet control performance on the search task, we encounter a difficulty. Control performance on the search task for the same frame condition is lower than control performance obtained in successive frame conditions and is lower than both control performance points for the adjacent targets condition. This finding suggests that when subjects were in the $100 \%$ search condition, the occurrence of the orientation symbol in another display position during the same frame was distracting. If we use the other three control conditions as possibly more appropriate estimates of letter search control performance, we find that the intersection of the "same frame" POC with search con- trol performance results in an estimate of orientation $\mathrm{d}^{\prime}$ of approximately zero.

In contrast, the intersection of the POC for successive frames with search control performance results in substantially above-chance performance of the orientation task. Presumably, in the successive frames condition, the subject can partially reallocate attention from the search task to the orientation task when they are separated in time. The separation employed here was evidently not large enough to allow complete reallocation of attention between the two tasks.

We suggest that both the visual search task and the orientation discrimination task are competing for a spatial attention mechanism and that performance of either of these tasks is close to chance if spatial attention is fully deployed to nontarget display positions.

This conclusion is supported by an examination of the POCs for the case in which both the orientation target and letter target were in adjacent display positions, shown in the right panel of Figure 2. First, consider the case of targets occurring in the same frame. All three dual-task conditions show that search performance is close to the level achieved in the $100 \%$ search condition. In fact, when subjects are emphasizing search, as in the $80 / 20$ condition $(80 \%$ attention to the search task and $20 \%$ to orientation discrimination), their search performance is slightly better than control performance. Partial attention to the orientation task evidently allows its position in space to bias the starting point of the letter search, and, when both targets are in adjacent positions, this bias is advantageous. As the subject shifts attention to the orientation task, this position advantage is partially offset by increased sharing of attention between the two discriminations.

Moving the orientation symbol to the frame following the search array had the effect of shifting the POC up and to the left in the case of adjacent tar-

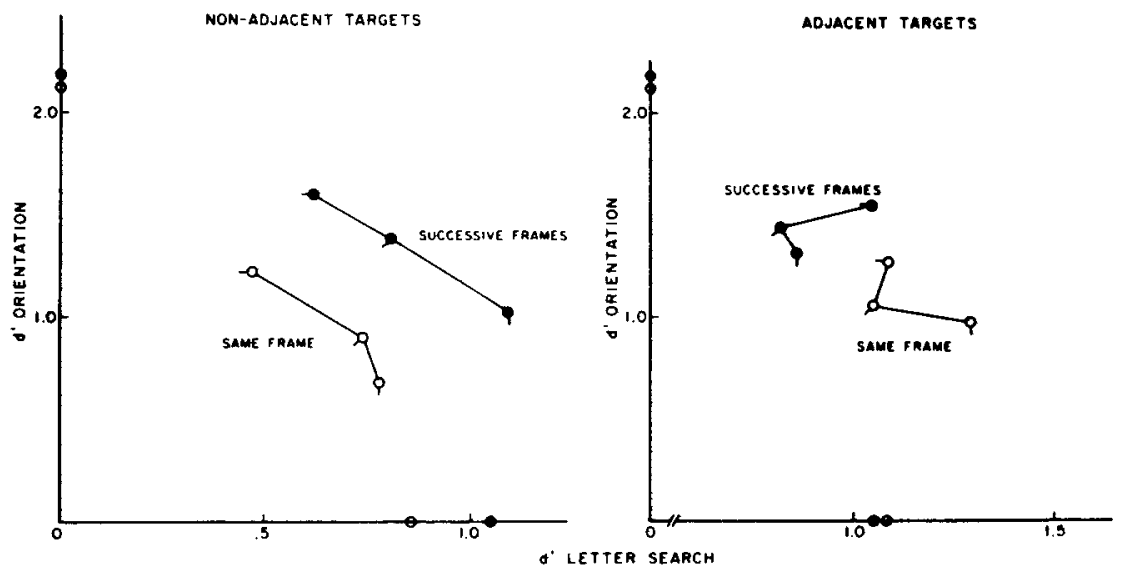

Figere 2. Performance opernting charneteristics for the case in which the target letter and orkentation form oceurred in nonadjacent and adjacent dispaly positions. 
gets. An upward shift indicates an improvement in orientation discrimination performance with temporal separation as occurred for nonadjacent targets. However, the leftward shift indicates decreased performance on the search task. This is clearly not a metacontrast effect of the after-occurring orientation form on the search target letter, because it does not occur in the $100 \%$ search condition. A more likely explanation is that the position-biasing effect of the orientation form on the search process is not as effective when it occurs after the array has been masked, just as delaying a partial report cue leads to a decline in identification of letters in iconic memory (Sperling, 1960).

The shape of the POC is instructive in this regard. When the subject is attending primarily to the letter search task (80/20 condition), performance on letter search in the adjacent targets/successive frames condition is slightly worse than the corresponding performance in the nonadjacent targets condition. In other words, when the subject is concentrating on the search task, there is no advantage in having attention drawn to the target letter position after the letter array has been masked. If we now consider corresponding points for the case of the subject attending primarily to the orientation information $(20 / 80$ condition), we see a substantial advantage for the case of adjacent targets relative to nonadjacent targets. In fact, the POC for adjacent targets "bends around" so that search performance is better in the $20 / 80$ condition than in the $80 / 20$ condition. This suggests that when the subject is concentrating on the orientation information, the allocation of spatial attention is "keyed" to the occurrence of the U-form. If this information does not occur until the frame following the letter array, the letter frame is held in a visual memory. This memory is probably posticonic but visual in nature. When the orientation symbol occurs next to the target letter, it aids the "readout" of information in that area.

We suggest that the memory for the display is a visual, posticonic one for several reasons. It appears to be visual because it is clearly preserving the positional information of the array letters. We suspect it is posticonic because the letter masks should have made it very difficult to read information from iconic memory. Several other authors have postulated a visual memory that is intermediate between iconic and long-term visual memory (Sperling \& Reeves, 1980; Turvey, 1978).

A repeated measures analysis of variance revealed that the effects described above were reliable. For search performance, both the main effect of adjacent vs. nonadjacent targets $[F(1,3)=283, p<.001]$ and its interactions with instructional condition $[F(3,9)=$ $6.06, p<.025]$ were significant. The effect of same vs. successive frames interacted significantly with whether or not targets were adjacent $[F(1,3)=18.3$, $p<.025]$, while the main effect of same/successive frames was not significant $[F(1,3)<1]$.

For orientation discrimination, both the main effects of instructions $[F(3,9)=29.7, p<.001]$ and same vs. successive frames $[F(1,3)=37.6, p<.001]$, as well as their interaction, were significant $[F(3,9)$ $=5.9, \mathrm{p}<.025]$. The last interaction is a bit misleading. As can be seen in Figure 2, the effects of these variables are almost perfectly additive, but the POCs are both approaching the same control performance level from different starting points.

\section{Contingency Analyses}

Recall that we were interested in measuring the spatial attention demands of visual search by observing the effect of target adjacency on the ability to discriminate the orientation of a briefly flashed form. The above analyses clearly show that letter search was improved when the orientation form occurred adjacent to the target letter relative to when it occurred in nonadjacent positions. The effect of the target letter position on orientation discrimination was, however, obscured in the above analyses. The position of the orientation form was always apparent even when the discrimination was incorrect. In contrast, any effects of position of the target letter should depend on whether the target was correctly detected. Consequently, we examined orientation discrimination contingent on the success of the letter search task.

Table 1 shows orientation discrimination contingent on correct and incorrect search. When search was correct, the orientation symbol was discriminated more accurately when it occurred adjacent to the target relative to when it occurred in nonadjacent positions $[F(1,3)=18.2, p<.025]$. Surprisingly, this effect was independent of whether the orientation symbol occurred in the same frame as the target or in the successive one $[F(1,3)<1]$.

When search was incorrect, the data suggest that the orientation symbol was discriminated better when it occurred in positions removed from the target letter. This effect just missed significance $[F(1,3)=$ $6.7, \mathrm{p}<.10]$. We suspect it is a real one, however,

Table 1

Proportion Correct Orientation Discrimination Contingent on Correct and Incorrect Search

\begin{tabular}{cccc}
\hline & & \multicolumn{2}{c}{ Location } \\
\cline { 3 - 4 } $\begin{array}{c}\text { Concurrent } \\
\text { Event }\end{array}$ & Frame & Adjacent & $\begin{array}{c}\text { Non- } \\
\text { Adjacent }\end{array}$ \\
\hline \multirow{2}{*}{ Search Correct } & Same & .61 & .53 \\
& Succ & .73 & .65 \\
Search Incorrect & Same & .50 & .57 \\
& Succ & .60 & .69 \\
\hline
\end{tabular}

Note-Same = same frame; Succ = successive frames. 
since all four subjects showed this pattern, although to varying degrees.

This pattern of results is consistent with a search process in which attention to the spatial position of the target is a key ingredient for successful performance. Correct detection of the target letter results in improved processing of other information in the same general area as the the target. When the target letter is not found, discrimination of material in the target area is suppressed relative to discrimination in other positions. These results are compatible with the conclusions derived from the POC analyses described earlier.

\section{DISCUSSION}

The goal of this experiment was to determine whether successful detection of a target letter in varied mapping visual search was dependent on spatial allocation of attention to the display region containing the target. This question was investigated by pairing the visual search task with a concurrent task of discriminating the orientation of a briefly flashed U-shaped target. This orientation symbol could occur in a position either adjacent or nonadjacent to the target letter.

Our results suggest that spatial attention to the target letter is a necessary component of successful search performance. The empirical performance operating characteristics (POCs) representing joint discrimination accuracy on the two tasks were quite different in the cases of adjacent and nonadjacent targets. When the target letter and orientation symbol occurred in different or nonadjacent display areas, there was an extensive tradeoff in performance of the two tasks. Indeed, when the letter array and orientation symbol occurred simultaneously, the POCs suggested that $100 \%$ attention to either task would result in near-chance performance of the other.

In contrast, when the orientation symbol occurred in the same frame and in the position adjacent to the target letter, search performance was close to the level obtained in the single-task control condition. Moving the orientation symbol to the succeeding frame actually decreased search performance for adjacent targets, indicating that the orientation symbol was less effective in manipulating attention to an object that had been masked.

Successful discrimination of the target letter also resulted in an increase in orientation discrimination accuracy for adjacent targets relative to the case in which they were nonadjacent. This relation was reversed when the subject failed to accurately detect the target. These results indicate that successful detection of a target letter is associated with attention to the spatial region of the target and, in addition, that errors in detection are associated with attention's being deployed to display regions not containing the target.
One way to conceptualize these results is as follows. Suppose that there is a limited visual processing resource that can be spread "thinly" over a wide area or concentrated in a restricted area. When there is spatial uncertainty concerning the location of targets, as in the present study, the subject begins the trial with attention in a "distributed state." In the case of letter search, we assume that the subject begins to accumulate information in parallel from each letter concerning the likelihood that it is a target. When the information in a particular location is sufficiently high to suggest the presence of a target, attention is allocated to that position, resulting in better processing of information in the target area and reduced processing of information in other areas (Shaw, 1978; Shaw \& Shaw, 1977).

In dual-task conditions, we assume that the allocation of visual attention can be triggered by either one of two events: the occurrence of the orientation symbol or the information accumulation process described above. The different attentional instructions used in the present study serve to determine the priority of these two different triggering mechanisms. Increasing the emphasis on one task increases the likelihood that it is that task that will control the allocation of attention. When the critical information for both tasks is located in the same area, there is less of a tradeoff in performance because either task can at least partially share the attention triggered by the other. In contrast, when the targets are in different areas, the attentional field cannot be shared and targets must be dealt with sequentially. According to this model, search errors result when a nontarget letter triggers an attention shift. This results in a withdrawal of attention from other areas of the display and would produce higher accuracy in discriminating the orientation symbol when it occurred in nonadjacent display positions, in agreement with the results of the present study.

Why invoke the notion of limited capacity, especially in view of the success of recent models that attribute all attentional limitations to memory and decision processes (cf. Duncan, 1980; Eriksen \& Spencer, 1969; Hoffman, 1978, 1979; Schneider \& Shiffrin, 1977; Shiffrin, 1978)? In considering this issue, it would first be useful to have a general characterization of late selection models.

Models that assume no limitation in "early" processing of the signal usually take the following form. Each letter in the display is represented by a random variable reflecting the likelihood that the letter is a target. The mean and variance of this random variable are independent of the attention that subjects allocate to its spatial position as well as of the number of other simultaneous inputs (Eriksen \& Spencer, 1969; Hoffman, 1978, 1979; Kinchla, 1974). The effect of attentional instructions may be to differentially weight these inputs when they are combined for the 
final decision (Kinchla, 1974). Alternatively, these signals may decay or be masked by subsequent input if they must enter a limited-capacity decision system in a serial manner (Duncan, 1980; Schneider \& Shiffrin, 1977). Either way, the effect of designating the spatial position of the target is to give it an advantage at the decision level relative to other competing inputs.

This approach faces difficulty in explaining any advantages in spatial allocation of attention to a target when it is the only form presented. Shaw and Shaw (1977) showed that recognition accuracy of a single letter was affected by the spatial allocation of attention to its position in space. Bashinski and Bacharach (1980) report a similar finding for a visual detection task. Similar effects for recognition latency were found by Eriksen and Hoffman (1974) and for detection latency by Posner, Snyder, and Davidson (1980). Unless one supposes that empty display positions are providing noise to a central decision process, these results seem to be strong evidence for an "early" effect of attention.

Notice that in the present study a similar advantage of spatial allocation of attention was observed. The discrimination of the orientation symbol was improved when it occurred in a position to which the subject was attending relative to the discrimination obtained for unattended positions. The shapes of the POCs as well as the results of the contingency analyses indicated that a component of processing the orientation symbol was attending to its position in space even though the display contained no other symbols that would be confusable with the orientation form. It is probably the case that both discriminations (search and orientation) are competing for a limited-capacity decision mechanism. If this were the only source of interference, however, we would not have observed the strong spatial dependencies between task performances observed in the present study.

Is it possible to account for our results within a framework that does not include a role for spatial attention? For example, suppose that in the letter discrimination task, the subject submits display letters in a serial fashion to working memory for comparison with the memory set. The effect of the orientation symbol may be to bias the order in which display letters enter working memory. In this interpretation, when the orientation symbol occurs adjacent to the target letter, it improves letter discrimination simply because the target letter reaches the comparison stage before its display representation is degraded by masking. This explanation would account for the improvement of search performance that occurred when the orientation symbol was adjacent to the target letter and is compatible with "late selection" models (Schneider \& Shiffrin, 1977).

The late selection model, however, would not predict an improvement of discrimination for the orientation symbol when it occurred adjacent to the target letter. Here there is only one input waiting for entry into working memory, and spatial attention cannot bias a choice between competing inputs. It seems more parsimonious to conclude that successful detection of a target letter involves attending to the position of the target letter and that one effect of attending to a spatial position is to improve the acuity for other forms in the same area.

\section{Related Work}

Our experiment is quite similar to experiments conducted by Treisman and Geffen (1967) and Treisman and Riley (1969). They asked subjects who were engaged in a shadowing task to also detect target words that could occur either in the shadowed or unshadowed message. They found that targets were better detected when they occurred in the shadowed message, in agreement with our results for visual "messages" occurring in the same spatial location.

This experiment is also similar to others that have attempted to measure the spatiotemporal distribution of visual attention (Eriksen \& Hoffman, 1972; Posner et al., 1980; Shulman, Remington, \& McLean, 1979). Our results, together with these previous studies, demonstrate that visual attention is a resource that can be distributed in space to differentially affect latency and accuracy of basic recognition and detection processes.

\section{Conclusion}

The successful detection of a target letter in a visual array is associated with allocation of attention to the spatial region of the target. Forms that occur within this attentional field are better discriminated than forms occurring elsewhere in the display. The ability to process simultaneous visual signals depends crucially on their relative locations in space.

\section{REFERENCE NOTE}

1. Sternberg, S. Scanning a persisting visual image vs. a memorized list. Paper presented at the annual meeting of the Eastern Psychological Association, Boston, April 1967.

\section{REFERENCES}

Bashinski, H. S., \& Bacharach, V. R. Enhancement of perceptual sensitivity as the result of selectively attending to spatial locations. Perception \& Psychophysics, 1980, 28, 241-248.

Colegate, R. L., Hoffman, J. E., \& Eriksen, C. W. Selective encoding from multielement visual displays. Perception \& Psychophysics, 1973, 14, 217-224.

Duncan, J. The locus of interference in the perception of simultaneous stimuli. Psychological Review, 1980, 87, 272-300.

ERIKSEN, B. A., \& Eriksen, C. W. Effects of noise letters upon the identification of a target letter in a nonsearch task. Perception \& Psychophysics, 1974, 16, 143-149.

Eriksen, C. W., \& Hoffman, J. E. Temporal and spatial characteristics of selective encoding from visual displays. Perception \& Psychophysics, 1972, 12, 201-204. 
Eriksen, C. W., \& Spencer, T. Rate of information processing in visual perception: Some results and methodological considerations. Journal of Experimental Psychology Monograph, 1969, 79 (2, Pt. 2).

Estes, W. K., \& TAYlor, H. A. Visual detection in relation to display size and redundancy of critical elements. Perception \& Psychophysics, 1966, 1, 9-16.

Hormman, J. E. Hierarchical stages in the processing of visual information. Perception \& Psychophysics, 1975, 18, 348-354.

Hofrman, J. E. Search through a sequentially presented visual display. Perception \& Psychophysics, 1978, 23, 1-11.

Hofrman, J. E. A two-stage model of visual search. Perception \& Psychophysics, 1979, 25, 319-327.

KINchlA, R. A. Detecting target elements in multielement arrays: A confusability model. Perception \& Psychophysics, 1974, 15, 149-158.

Kinchla, R. A. The measurement of attention. In R. S. Nickerson (Ed.), Attention and performance VIII. Hillsdale, N.J: Erlbaum, 1980.

LAppin, J. S., \& UtTal, W. R. Does prior knowledge facilitate the detection of visual targets in random noise? Perception \& Psychophysics, 1976, 20, 365-374.

Navon, D., \& Gopher, D. On the economy of the humanprocessing system. Psychological Review, 1979, 86, 214-255.

Posner, M. I., Snyder, C. R. R., \& Davidson, B. J. Attention and the detection of signals. Journal of Experimental Psychology: General, 1980, 109, 160-174.

Schneidea, W., \& Shifrrin, R. M. Controlled and automatic human information processing: I. Detection, search, and attention. Psychological Review, 1977, 84, 1-66.

ShAw, M. L. A capacity allocation model for reaction time. Journal of Experimental Psychology: Human Perception and Performance, 1978, 4, 586-598.

Shaw, M., \& Shaw, A. Optimal allocation of cognitive resources to spatial locations. Journal of Experimental Psychology: Human Perception and Performance, 1977, 3, 201-211.

Shiffrin, R. M. Capacity limitations in information processing, attention, and memory. In W. K. Estes (Ed.), Handbook of learning and cognitive processes (Vol. 4). Hillsdale, N.J: Erlbaum, 1978.

Shiffrin, R. M., \& Gardner, G. T. Visual processing capacity and attentional control. Journal of Experimental Psychology, $1972,93,72-82$.

Shulman, G. L., Remington, R. W., \& Mclean, J. P. Moving attention through space. Journal of Experimental Psychology: Human Perception and Performance, 1979, 5, 522-526.

SPERLing, G. The information available in brief visual presentations. Psychological Monographs, 1960, 74(11, Whole No. 498).

Sperling, G., \& Melchner, M. J. The attention operating characteristic: Examples from visual search. Science, 1978, 202, 315-318.

Sperling, G., \& Reeves, A. Measuring the reaction time of a shift of visual attention. In R. S. Nickerson (Ed.), Attention and performance VIII. Hillsdale, N.J: Erlbaum, 1980.

Treisman, A. M., \& Geffen, G. Selective attention: Perception or response? Quarterly Journal of Experimental Psychology, $1967,19,1-17$.

Treisman, A. M., \& Riley, R. G. Is selective attention selective perception or selective response? A further test. Journal of Experimental Psychology, 1969, 79, 27-34.

TuRveY, M. T. Visual processing and short-term memory. In W. K. Estes (Ed.), Handbook of learning and cognitive processes (Vol. 5). Hillsdale, N.J: Erlbaum, 1978.

\section{NOTE}

1. A preliminary analysis of positions that were nonadjacent to the target letter failed to reveal any systematic differences in the three nonadjacent positions. Consequently, they were averaged to produce the "nonadjacent" category.

(Manuscript received January 16, 1981; revision accepted for publication June 16,1981 .) 